\title{
A class of complete arcs in multiply derived planes
}

\author{
Arrigo Bonisoli and Gloria Rinaldi
}

Al Professore Adriano Barlotti

\begin{abstract}
We prove that unital-derived $\left(q^{2}-q+1\right)$-arcs of $\mathrm{PG}\left(2, q^{2}\right)$ still yield complete arcs after multiple derivation with respect to disjoint derivation sets on a given line.
\end{abstract}

\section{Introduction}

There is currently limited knowledge of complete arcs in non-desarguesian planes. Excluding ovals and hyperovals, few constructions are known for "infinite" families of planes. Many constructions for non-desarguesian planes involve suitable replacement of some desarguesian lines. For any given arc in the original desarguesian plane the question arises whether it remains an arc in the new plane. For a complete arc of the original plane which remains an arc after line replacement the additional question is whether completeness is also maintained in the new plane: we refer to the introduction of the paper [13] for an account of results in this area.

The so called "unital-derived" arcs of $\mathrm{PG}\left(2, q^{2}\right)$ were first constructed by B. Kestenband [9] and were then studied by various authors from different points of view [10], [2], [5], [4]. They are complete $\left(q^{2}-q+1\right)$-arcs in $\operatorname{PG}\left(2, q^{2}\right)$ and it was proved in [11] that they yield arcs in the Hall plane of the same order. Their completeness in the Hall plane is guaranteed whenever $q^{2}>9$; in the Hall plane of order 9 these arcs can be completed by the adjunction of at most one point, see [12].

A Hall plane can be viewed as obtained from the corresponding desarguesian plane by a single derivation. From this standpoint we can say that we control the behaviour of unital-derived arcs under single derivation. It seems quite natural to try to see what happens if we derive more than once. It is the purpose of the present paper to prove that the unital-derived arcs of $\mathrm{PG}\left(2, q^{2}\right)$ remain complete arcs when multiple derivation is performed with respect to disjoint derivation sets on a given line.

How large are these arcs in comparison with other complete arcs in these or other planes? We do not address this problem here.

\section{Background results}

For a prime power $q$ denote by $\pi$ the desarguesian projective plane $\operatorname{PG}\left(2, q^{2}\right)$. We denote by ${ }^{t} X=(x, y, z)$ a point of $\pi$. For an arbitrary matrix $M$ with entries 
in $\mathrm{GF}\left(q^{2}\right)$ we denote by $\bar{M}$ the matrix obtained from $M$ by raising each entry to the $q$-th power.

For each non-degenerate $3 \times 3$ hermitian matrix $H$ over $\operatorname{GF}\left(q^{2}\right)$, the $q^{3}+1$ points $X$ satisfying the equation ${ }^{t} X H \bar{X}=0$ are the points of a hermitian unital which is embedded in $\pi$. We shall denote this classical unital by $\{H\}$ and we shall often identify $\{H\}$ with its point-set.

Fix a line at infinity $\ell_{\infty}$ in $\pi$ and let $S^{1}, \ldots, S^{r}, r \leqslant q-1$, be disjoint derivation sets for $\pi$ on $\ell_{\infty}$. Since all derivation sets $S^{1}, \ldots, S^{r}$ lie on a given line, deriving $\pi$ with respect to $S^{1}, \ldots, S^{r}$ one obtains a plane $\pi_{S^{1}, \ldots, S^{r}}$ of order $q^{2}$, which is an André plane, [7, Theorem 10.12]. The affine points of this new plane are the affine points of $\pi$ and the affine lines are the lines of $\pi$ not meeting $S^{1} \cup \cdots \cup S^{r}$, together with the Baer subplanes of $\pi$ which contain $S^{i}$ for some index $i=1, \ldots, r$. Furthermore, the derived plane $\pi_{S^{i}}$ is a Hall plane [7, corollary to Theorem 10.13].

Let $U$ be a hermitian unital of $\pi$. Theorem 1.1 in [1] shows that there are five possible relationships between $U$ and a derivation set $S^{i}$. More precisely:

(a) $U \cap \ell_{\infty}=S^{i}$, then the point set of $U$ yields a Buekenhout unital $U^{\prime}$ in $\pi_{S^{i}}$, [6].

(b) $U$ is secant to $\ell_{\infty}$ and $S^{i} \cap U=\varnothing$, then the point set of $U$ yields a Buekenhout unital $U^{\prime}$ in $\pi_{S^{i}}$.

(c) $U$ is secant to $\ell_{\infty}$ with $0<\left|S^{i} \cap U\right|<q+1$, then the point-set of $U$ does not yield a unital in $\pi_{S^{i}}$.

(d) $U$ is tangent to $\ell_{\infty}$ with $U \cap \ell_{\infty} \subseteq S^{i}$, then the point-set of $U$ does not yield a unital in $\pi_{S^{i}}$.

(e) $U$ is tangent to $\ell_{\infty}$ and $U \cap \ell_{\infty} \nsubseteq S^{i}$, then the point-set of $U$ yields a BuekenhoutMetz unital $U^{\prime}$ in $\pi_{S^{i}}$.

Each line of the multiply derived plane $\pi_{S^{1}, \ldots, S^{r}}$ is a line of $\pi_{S^{i}}$ for some $S^{i}$. A unital $U$ of $\pi$ yields a unital $U^{\prime}$ in the multiply derived plane $\pi_{S^{1}, \ldots, S^{r}}$ precisely when one of the following holds:

(i) $U$ is secant to $\ell_{\infty}$ and $S^{i}=U \cap \ell_{\infty}$, for some index $i=1, \ldots, r$.

(ii) $U$ is secant to $\ell_{\infty}$ and $S^{i} \cap U=\varnothing$, for every index $i=1, \ldots, r$.

(iii) $U$ is tangent to $\ell_{\infty}$ and $U \cap \ell_{\infty} \nsubseteq S^{1} \cup \cdots \cup S^{r}$.

In fact since $U$ consists of $q^{3}+1$ points of $\pi$, it also yields as many points in $\pi_{S^{1}, \ldots, S^{r}}$. It is then sufficient to observe that, if the line joining two given points of $U$ in $\pi_{S^{1}, \ldots, S^{r}}$ meets some derivation set $S^{i}$, then it is a line of the Hall plane $\pi_{S^{i}}$ and then apply (a), (b) to obtain that this line intersects $U$ in $q+1$ points; if it meets no such derivation set, then this desarguesian line remains unaltered in the derivation process and so it intersects $U$ in $q+1$ points.

Considering the common intersection of $q+1$ suitably chosen hermitian unitals of $\pi$, a class of $\left(q^{2}-q+1\right)$-arcs of $\pi$ was constructed by B. C. Kestenband, [9]. These arcs are referred to as unital-derived arcs and their completeness in $\pi$ was proved with different methods, [2], [4], [5]. 
It was proved in [11] that the unital-derived arcs of $\pi$ remain arcs in the Hall plane $\pi_{S^{i}}$ of the same order, provided that the derivation set is suitably chosen. Furthermore, their completeness in the Hall plane was proved whenever $q^{2}>9$. The case of the Hall plane of order 9 is considered in detail in [12]: in this case the arcs are either complete or can be completed by adding exactly one point.

The proof of these results requires some properties of the unital-derived arcs of $\pi$, some of which are briefly summarized here. Let $H$ be a non-degenerate $3 \times 3$ hermitian matrix over $\operatorname{GF}\left(q^{2}\right)$ whose characteristic polynomial is irreducible over $\operatorname{GF}\left(q^{2}\right)$, or, which is the same, over $\mathrm{GF}(q)$. If $\lambda \in \mathrm{GF}(q)$ and $r, s$ are distinct indices in $\left\{0,1, \ldots, q^{2}+q\right\}$ then $H^{s}-\lambda H^{r}$ is itself a rank 3 hermitian matrix and the point-set $\left\{H^{r}\right\} \cap\left\{H^{s}\right\}$ is a complete unital-derived arc obtained as the common intersection of the $q+1$ hermitian unitals $\left\{H^{r}\right\},\left\{H^{s}-\lambda H^{r}\right\}, \lambda \in \mathrm{GF}(q)$.

Furthermore, the plane $\pi$ is the disjoint union of $q^{2}+q+1$ unital-derived arcs and each such arc is the point-orbit of a subgroup of a Singer cycle of $\pi,[4],[10]$, [8]. It is also proved in [10], [8], that the unitals under consideration and their intersections can be viewed as the lines and points of a projective plane $\operatorname{PG}(2, q)$.

\section{Arcs in multiply derived planes}

Let $g(x)=-x^{3}+s x^{2}+n x+p \in \mathrm{GF}(q)[x]$ be a cubic polynomial which is irreducible over $\mathrm{GF}(q)$ and hence also over $\mathrm{GF}\left(q^{2}\right)$. Fix distinct elements $a, b \in \mathrm{GF}(q)$ and define $c=s-(a+b)$. Determine $e, f \in \mathrm{GF}\left(q^{2}\right)$ satisfying the following equations:

$$
\begin{gathered}
e^{q+1}+f^{q+1}=n+a b+a c+b c \\
-b e^{q+1}-a f^{q+1}=p-a b c
\end{gathered}
$$

We observe incidentally that if the elements $e, f$ satisfy the previous equations, then so do the elements $e^{q}, f$.

The matrices

$$
H=\left(\begin{array}{lll}
a & 0 & e \\
0 & b & f \\
e^{q} & f^{q} & c
\end{array}\right), \quad \hat{H}=\left(\begin{array}{ccc}
a & 0 & e^{q} \\
0 & b & f \\
e & f^{q} & c
\end{array}\right)
$$

are easily seen to be rank 3 hermitian matrices with $g(x)$ as characteristic polynomial.

Let $\mathscr{A}$ be the unital derived-arc of $\pi$ obtained as the common intersection of the $q+1$ unitals $\{I\},\{H-\lambda I\}, \lambda \in \mathrm{GF}(q)$. Let $\hat{\mathscr{A}}$ be the unital derived-arc of $\pi$ obtained as the common intersection of the $q+1$ unitals $\{I\},\{\hat{H}-\lambda I\}, \lambda \in \mathrm{GF}(q)$.

We define $\ell_{\infty}$ to be the line of $\pi$ with equation $z=0$. Let $t \in \mathrm{GF}(q)^{*}$ and denote by $S_{t}$ the point-set on $\ell_{\infty}$ consisting of all points $(1, m, 0)$ with $m^{q+1}=t$. The set $S_{t}$ is a derivation-set for $\pi$ on $\ell_{\infty}$, [7, Theorem 10.11]. The unital $\{I\}$ intersects $\ell_{\infty}$ in $S_{-1}$, while each unital $\{H-\lambda I\}$ (as well as each unital $\{\hat{H}-\lambda I\}$ ) with $\lambda \neq a$, $\lambda \neq b$ intersects $\ell_{\infty}$ in $S_{(\lambda-a) /(b-\lambda)}$. Finally, we have $\ell_{\infty} \cap\{H-a I\}=\ell_{\infty} \cap\{\hat{H}-a I\}=$ $\{(1,0,0)\}, \ell_{\infty} \cap\{H-b I\}=\ell_{\infty} \cap\{\hat{H}-b I\}=\{(0,1,0)\}$ and $\mathscr{A} \cap \ell_{\infty}=\hat{\mathscr{A}} \cap \ell_{\infty}=\varnothing$. 
We choose $r$ distinct elements $t_{1}, \ldots, t_{r}$ in $\operatorname{GF}(q)^{*}=\{-1\} \cup\{(\lambda-a) /(b-\lambda)$ : $\lambda \in \mathrm{GF}(q)-\{a, b\}\}$. We denote by $\pi_{1, \ldots, r}$ the multiply derived plane $\pi_{S_{t_{1}}, \ldots, S_{t_{r}}}$ and by $\hat{\pi}_{1, \ldots, r}$ the multiply derived plane corresponding to the derivation sets $S_{t}$ where $t$ varies in $\mathrm{GF}(q)^{*}-\left\{t_{1}, \ldots, t_{r}\right\}$.

Observe that this kind of multiple derivation is only meaningful for $r<q-1$, since deriving $\mathrm{PG}\left(2, q^{2}\right)$ with respect to $q-1$ pairwise disjoint derivation sets on $\ell_{\infty}$ gives rise to a Desarguesian plane again, [7, Exercise 10.4].

\section{Proposition 1. The set $\mathscr{A}$ is an arc in the multiply derived plane $\pi_{1, \ldots, r}$.}

Proof. The previous section shows that the $q+1$ unitals mutually intersecting in $\mathscr{A}$ are still unitals in $\pi_{1, \ldots, r}$ and they cover the points of the plane. If a line meets $\mathscr{A}$ in $h \geqslant 2$ points, the same line intersects each of the $q+1$ unitals in $q+1-h$ points outside $\mathscr{A}$. Therefore $(q+1)(q+1-h)+h=q^{2}+1$, whence $h=2$.

Proposition 2. If $r<(q-1)(q-2) /(q+1)$ then the arc $\mathscr{A}$ is complete in the multiply derived plane $\pi_{1, \ldots, r}$.

Proof. We begin by proving the completeness of $\mathscr{A}$ on $\ell_{\infty}$.

Let $j$ be the involution on the points of $\pi$ defined as follows: $j(x, y, 1)=\left(x^{q}, y, 1\right)$; $j(x, y, 0)=(x, y, 0)$. The point-set $j(\mathscr{A})$ is precisely the unital-derived arc obtained as the common intersection of $\{I\}$ and $\{\hat{H}-\lambda I\}, \lambda \in \mathrm{GF}(q)$.

Since $\mathscr{A} \cap \ell_{\infty}=\varnothing$ and $\mathscr{A}$ is a complete arc in the desarguesian plane $\pi$, when considering a possible candidate for adjunction in the multiply derived plane we only need to consider a point $P_{\infty}=(1, m, 0)$, with $P_{\infty} \in S_{t_{i}}$, for some $i \in\{1, \ldots, r\}$. The arc $j(\mathscr{A})$ is complete in the desarguesian plane $\pi$. We find thus an element $v \in \operatorname{GF}\left(q^{2}\right)$ and a line $\ell$ with equation $y=m x+v$ having two points in common with $j(\mathscr{A})$. Therefore, the set $j(\ell)$ has 2 points in common with $\mathscr{A}$. In the multiply derived plane, the set $j(\ell)$ is the point-set of the line with equation $y=m x^{q}+v$ which contains $P_{\infty}$. This proves the completeness of $\mathscr{A}$ on the line at infinity.

Now we prove the completeness on the affine points of $\pi_{1, \ldots, r}$.

Let $P$ be an affine point and suppose $\mathscr{A} \cup\{P\}$ is still an arc of $\pi_{1, \ldots, r}$. Each line of $\pi_{1, \ldots, r}$ passing through $P$ has thus either 0 or 1 point in $\mathscr{A}$. The arc $\mathscr{A}$ is complete in $\pi$, so each line of $\pi$ which contains $P$ and has 2 points in $\mathscr{A}$ must intersect $\ell_{\infty}$ at a point of $S_{t_{i}}$, for some $i$. Consider all lines of $\pi$ passing through $P$ which are 2-secants of $\mathscr{A}$. Let $\mathscr{K} \subset \mathscr{A}$ be the subset consisting of all points in $\mathscr{A}$ lying on one such line and denote by $k$ the cardinality of $\mathscr{K}$, an even number.

The plane $\pi$ is the disjoint union of $q^{2}+q+1$ complete unital-derived arcs, [8], one of which is $\mathscr{A}$. Let $\mathscr{B}$ be the unital-derived arc containing the point $P$. Let $T$ be a fixed point of the set $\mathscr{A}-\mathscr{K}$. There are $q^{2}+1$ lines of $\pi$ containing $T$. The condition $|\mathscr{A}|=q^{2}-q+1$ implies that exactly $q^{2}-q$ of these lines are 2 -secants of $\mathscr{A}$ and exactly $q+1$ lines of $\pi$ are tangent to $\mathscr{A}$ at $T$; these lines may or may not contain points of $\mathscr{B}$.

Now, the lines which are tangent to $\mathscr{A}$ at $T$ and contain points of $\mathscr{B}$ are at least $\left(q^{2}-q-k\right) / 2+1$. In fact, the unital-derived arcs partitioning $\pi$ are the orbits of 
a cyclic collineation group, generated by, say, $\theta$. Let $S \in \mathscr{A}-\mathscr{K}, S \neq T$, and let $\theta^{i}(S)=T$. We have $\theta^{i}(P)=Q$, where $Q$ is also a point of $\mathscr{B}$ and the line $Q T$ is tangent to $\mathscr{A}$ because so is the line $P S$. Observe that the line $Q T$ is either a tangent or a 2-secant of $\mathscr{B}$. Assume $Q T$ intersects $\mathscr{B}$ in $Q$ and $Q^{\prime}$ and write $\theta^{j}(P)=Q^{\prime}, \theta^{i} \neq \theta^{j}$, and $\theta^{i}(S)=T, \theta^{j}\left(S^{\prime}\right)=T$. As $S$ varies in $\mathscr{A}-\mathscr{K}$, the line $Q T$ is counted twice. Therefore, the number of lines which are tangent to $\mathscr{A}$ at $T$ and contain points of $\mathscr{B}$ is at least $\left[\frac{|\mathscr{A}-\mathscr{K}|}{2}\right]$ and since $|\mathscr{A}-\mathscr{K}|=q^{2}-q+1-k$ is odd, we see that this number is at least $\left(q^{2}-q-k\right) / 2+1$.

Furthermore, $\mathscr{A}$ and $\mathscr{B}$ are contained in a unique unital $U$ of $\pi$, hence the tangent line to $U$ at $T$ is also tangent to $\mathscr{A}$ at $T$ and this line has no points in common with $\mathscr{B}$. Altogether, we have at least $\left(q^{2}-q-k\right) / 2+2$ lines that are tangent to $\mathscr{A}$ at $T$. We know this number to be less than or equal to $q+1$ and the relation $\left(q^{2}-q-k\right) / 2+2 \leqslant q+1$ is equivalent to $q^{2}-3 q+2 \leqslant k$.

Let $\mathscr{K}=\left\{P_{i} \mid i=1, \ldots, k\right\}$. We are supposing $\mathscr{A} \cup\{P\}$ to be an arc in $\pi_{1, \ldots, r}$. Therefore each line $P P_{i}$ of $\pi$ contains a point of some derivation set $S_{t_{i}}$. Furthermore, the line of $\pi_{1, \ldots, r}$ containing $P$ and $P_{i}$ is the unique Bear subplane of $\pi$ containing $P, P_{i}$ and the derivation set $S_{t_{i}}$. This plane has no points in common with $\mathscr{A} \cup\{P\}$ other than $P$ and $P_{i}$. We obtain thus $k$ distinct Bear subplanes which contain $P$ and have no other affine point in common. These $k$ Baer subplanes are lines of $\pi_{1, \ldots, r}$ corresponding to distinct points of $\ell_{\infty}$. We obtain thus at most $r(q+1)$ points at infinity and we have the relation $q^{2}-3 q+2 \leqslant k \leqslant r(q+1)$, contradicting $r<$ $(q-1)(q-2) /(q+1)$.

Proposition 3. If $q>3$ the arc $\mathscr{A}$ is complete in the multiply derived plane $\pi_{1, \ldots, r}$.

Proof. We know by the previous proposition that if $r<(q-1)(q-2) /(q+1)$ then the arc $\mathscr{A}$ is complete. We assume thus $r \geqslant(q-1)(q-2) /(q+1)$. The mapping

$$
(x, y) \mapsto\left(x^{q}, y\right), \quad(m) \mapsto(m), \quad(\infty) \mapsto(\infty)
$$

yields an isomorphism from the plane $\pi_{1, \ldots, r}$ to the plane $\hat{\pi}_{1, \ldots, r}$, see [7, Exercise 10.4]. Furthermore, this isomorphism maps the point-set $\mathscr{A}$ of $\pi_{1, \ldots, r}$ to the point-set $\hat{\mathscr{A}}$ of $\hat{\pi}_{1, \ldots, r}$, and we already know that $\mathscr{A}$ and $\hat{\mathscr{A}}$ are arcs. We prove the completeness of $\mathscr{A}$ in $\pi_{1, \ldots, r}$ by proving the completeness of $\hat{\mathscr{A}}$ in $\hat{\pi}_{1, \ldots, r}$.

By Proposition 2 the arc $\hat{\mathscr{A}}$ will certainly be complete whenever the inequality $q-1-r<(q-1)(q-2) /(q+1)$ holds. It follows from $r \geqslant(q-1)(q-2) /(q+1)$ that the inequality $q-1-r \leqslant 3(q-1) /(q+1)$ also holds. If $q>5$ then we have $3(q-1) /(q+1)<(q-1)(q-2) /(q+1)$ and the assertion follows in this case. For $q=4$ or 5 and $r=1$ the completeness of $\mathscr{A}$ follows from Proposition 2. For $q=4$ and $r=2$ we still have $q-1-r<(q-1)(q-2) /(q+1)$ and the previous argument still applies. For $q=5$ and $r=3$ the completeness of $\hat{A}$ follows from Proposition 2 and so we also have the completeness of $\mathscr{A}$ as above. For $q=5$ and $r=2$ we may limit the choice of two derivation sets $S_{t_{1}}$ and $S_{t_{2}}$ to the cases $\left(t_{1}, t_{2}\right)=(-1,1),(-1,2)$ and $(-1,3)$ respectively. We performed calculations with 
the computer algebra system MAGMA [3] checking that the arc $\mathscr{A}$ is complete for all such choices: source code is available from the authors.

The request $q>3$ in the previous proposition is no restriction, since only single derivation is meaningful for $q=3$.

\section{References}

[1] S. G. Barwick, Unitals in the Hall plane. J. Geom. 58 (1997), 26-42. MR 98b:51013 Zbl 0893.51007

[2] E. Boros, T. Szönyi, On the sharpness of a theorem of B. Segre. Combinatorica 6 (1986), 261-268. MR 87m:51022 Zbl 0605.51008

[3] J. Cannon, W. Bosma, Handbook of MAGMA functions. University of Sydney 2001.

[4] A. Cossidente, A new proof of the existence of $\left(q^{2}-q+1\right)$-arcs in PG $\left(2, q^{2}\right)$. J. Geom. 53 (1995), 37-40. MR 96e:51009 Zbl 0839.51009

[5] J. C. Fisher, J. W. P. Hirschfeld, J. A. Thas, Complete arcs in planes of square order. In: Combinatorics '84 (Bari, 1984) Ann. Discr. Math. 30 (1986), 243-250. MR 87m:51025 Zbl 0589.51020

[6] K. Grüning, A class of unitals of order $q$ which can be embedded in two different planes of order $q^{2}$. J. Geom. 29 (1987), 61-77. MR 88j:51015 Zbl 0623.51002

[7] D. R. Hughes, F. C. Piper, Projective planes. Springer 1973. MR $48 \# 12278$ Zbl 0267.50018

[8] B. C. Kestenband, Projective geometries that are disjoint unions of caps. Canad. J. Math. 32 (1980), 1299-1305. MR 83h:51021 Zbl 0414.51001

[9] B. C. Kestenband, Unital intersections in finite projective planes. Geom. Dedicata 11 (1981), 107-117. MR 82h:05013 Zbl 0452.51007

[10] B. C. Kestenband, A family of complete arcs in finite projective planes. Colloq. Math. 57 (1989), 59-67. MR 9lb:51017 Zbl 0695.51011

[11] G. Rinaldi, Complete unital-derived arcs in the Hall planes. Abh. Math. Sem. Univ. Hamburg 71 (2001), 197-203. MR 2002j:51008 Zbl 01708581

[12] G. Rinaldi, F. Zironi, Complete unital-derived arcs in the Hall plane of order 9. Bull. Inst. Combin. Appl. 36 (2002), 29-36. MR 1936288

[13] T. Szönyi, Complete arcs in non-Desarguesian planes. Ars Combin. 25 (1988), 169-178. MR 89d:51013 Zbl 0652.51002

Received 26 November, 2002; revised 13 March, 2003

A. Bonisoli, Dipartimento di Scienze Sociali, Cognitive e Quantitative, Università di Modena e Reggio Emilia, via Giglioli Valle 9, 42100 Reggio Emilia, Italy

Email: bonisoli.arrigo@unimore.it

G. Rinaldi, Dipartimento di Scienze Agrarie, della Formazione Primaria e della Programmazione Sanitaria, Università di Modena e Reggio Emilia, via Kennedy 17, 42100 Reggio Emilia, Italy

Email: rinaldi.gloria@unimore.it 